\title{
Effects of Atropine and Gastric Inhibitory Polypeptide on Hepatic Glucose Uptake and Insulin Extraction in Conscious Dogs
}

\author{
Zvi Chap, Toshihiko lshida, Jesse Chou, Robert Lewis, Craig Hartley, Mark Entman, and James B. Field \\ Diabetes Research Laboratory, St. Luke's Episcopal Hospital, Division of Endocrinology and Metabolism, Baylor College of Medicine, \\ Houston, Texas 77030; and Section of Cardiovascular Sciences, Department of Medicine, \\ Baylor College of Medicine, Houston, Texas 77030
}

\section{Abstract}

Previous studies comparing the effects of oral, intraportal, and peripheral venous administration of glucose in conscious dogs demonstrated a significant increase in hepatic extraction of insulin only after oral glucose, but similar hepatic uptake of glucose after oral and intraportal glucose, which was greater than that after peripheral intravenous glucose infusion. This study evaluated the effect of atropine blockade of the parasympathetic nervous system on the increased fractional hepatic extraction of insulin and the role of gastric inhibitory polypeptide (GIP) on augmented hepatic uptake of oral glucose in conscious dogs with chronically implanted Doppler flow probes on the portal vein and hepatic artery, and catheters in the portal and hepatic veins and carotid artery. Since atropine infusion decreased absorption of glucose, and in order to achieve comparable portal vein levels of glucose and insulin, the dogs receiving atropine were given $1.9 \pm 0.1 \mathrm{~g} / \mathrm{kg}$ glucose, compared with the control dogs who received $1.1 \pm 0.1 \mathrm{~g} / \mathrm{kg}$. The percentage of the glucose load that was absorbed was greater in the dogs not given atropine $(80 \pm 4$ vs. $44 \pm 7 \%$ ), but because of the different loads, the absolute amount of glucose absorbed was similar in both groups $(20.2 \pm 1.6$ vs. $21.7 \pm 4.1 \mathrm{~g})$. Although delayed by atropine, the peak portal vein glucose and insulin concentrations and the amounts presented to the liver were similar in both groups. However, the increased portal vein plasma flow and fractional hepatic extraction of insulin observed after oral glucose was not observed in the dogs infused with atropine. The net hepatic glucose uptake after oral glucose was significantly less at 10, 20, and $45 \mathrm{~min}$ in the atropine-treated dogs, and the area under the curve over the 180-min period was $44 \%$ less. However, the latter was not statistically significant. Infusion of GIP with peripheral intravenous glucose did not increase hepatic uptake of glucose or the fractional hepatic extraction of insulin compared with peripheral intravenous glucose alone. These results indicate an important role for parasympathetic innervation in the augmented fractional hepatic extraction of insulin, and increased portal vein plasma flow after oral glucose. Although a relationship between the augmented fractional extraction of insulin and the net hepatic glucose uptake may exist, it does not necessarily indicate that the former is required for the latter. Such parasympathetic innervation may

Dr. Ishida's current address is First Department of Medicine, Kagawa Medical School, Miki, Kita, Kagawa 761-07, Japan. Address correspondence to Dr. Field, St. Luke's Episcopal Hospital.

Received for publication 28 December 1983 and in revised form 24 April 1985.

J. Clin. Invest.

(C) The American Society for Clinical Investigation, Inc.

0021-9738/85/09/1174/08 \$1.00

Volume 76, September 1985, 1174-1181 be involved in the greater removal of glucose by the liver after oral compared with peripheral glucose administration. The augmented hepatic uptake of glucose and fractional hepatic extraction of insulin after oral glucose does not appear to be mediated by gastric inhibitory polypeptide.

\section{Introduction}

The fractional hepatic extraction of insulin increased after the oral administration of glucose (1-3). Under these circumstances, both the amount of insulin and glucose presented to the liver also increased, but neither one seems to be the signal for the augmented fractional hepatic extraction of insulin. Thus, infusion of insulin into the portal circulation, which reproduced the concentrations achieved after oral glucose, did not increase fractional hepatic uptake of insulin (4). In addition, infusion of glucose into the portal vein to match the portal vein glucose concentration obtained after oral glucose did not augment fractional hepatic extraction of insulin (3). In these latter studies, the net hepatic uptake of glucose was similar whether the glucose was given orally or infused into the portal vein, confirming the earlier results of Bergman et al. (5). However, such net hepatic uptake of glucose was greater than when an equivalent amount of glucose was infused into a peripheral vein (3). The present studies examined the role of the parasympathetic nervous system on the augmented fractional hepatic extraction of insulin and hepatic glucose uptake after oral glucose. The possibility that gastric inhibitory polypeptide (GIP) ${ }^{1}$ was responsible for the greater hepatic glucose uptake after oral compared with peripheral intravenous glucose administration was tested by portal infusion of GIP and peripheral infusion of glucose.

\section{Methods}

Animals and surgery. Healthy, adult male and female mongrel dogs, weighing 20-36 kg, were prepared with catheters in the portal vein, left common hepatic vein, and carotid artery, and Doppler flow probes on the portal vein and hepatic artery as previously described (3). The tip of the catheter in the portal vein was positioned immediately below the portal vein bifurcation. Postoperatively, the dogs were fed one can of Ken-L Ration (Ralston Purina Co., St. Louis, MO) each day, and the catheters were flushed with $2 \mathrm{ml}$ heparinized saline $(50 \mathrm{U} / \mathrm{ml})$ daily to prevent thrombosis. Experiments were done in overnight fasted, conscious, and unrestrained animals at least 2 wk after recovery from surgery. Experiments were done on animals whose hematocrits were $>30 \%$, who appeared in healthy condition, and had a good appetite with normal stools. During the experiments, phasic and mean control blood pressure was measured using a Statham $\mathrm{P}^{23} \mathrm{db}$ pressure transducer that was connected to the arterial catheter. Except for an initial increase associated with ingestion of glucose, the blood pressure did not change significantly

1. Abbreviation used in this paper: GIP, gastric inhibitory polypeptide. 
throughout each experiment. Blood samples for glucose, insulin, glucagon, and GIP were collected simultaneously from the portal vein, hepatic vein, and carotid artery in chilled tubes containing $500 \mathrm{U}$ Trasylol (FBA Pharmaceuticals, Inc., New York, NY) and $1.2 \mathrm{mg}$ EDTA/ml of blood.
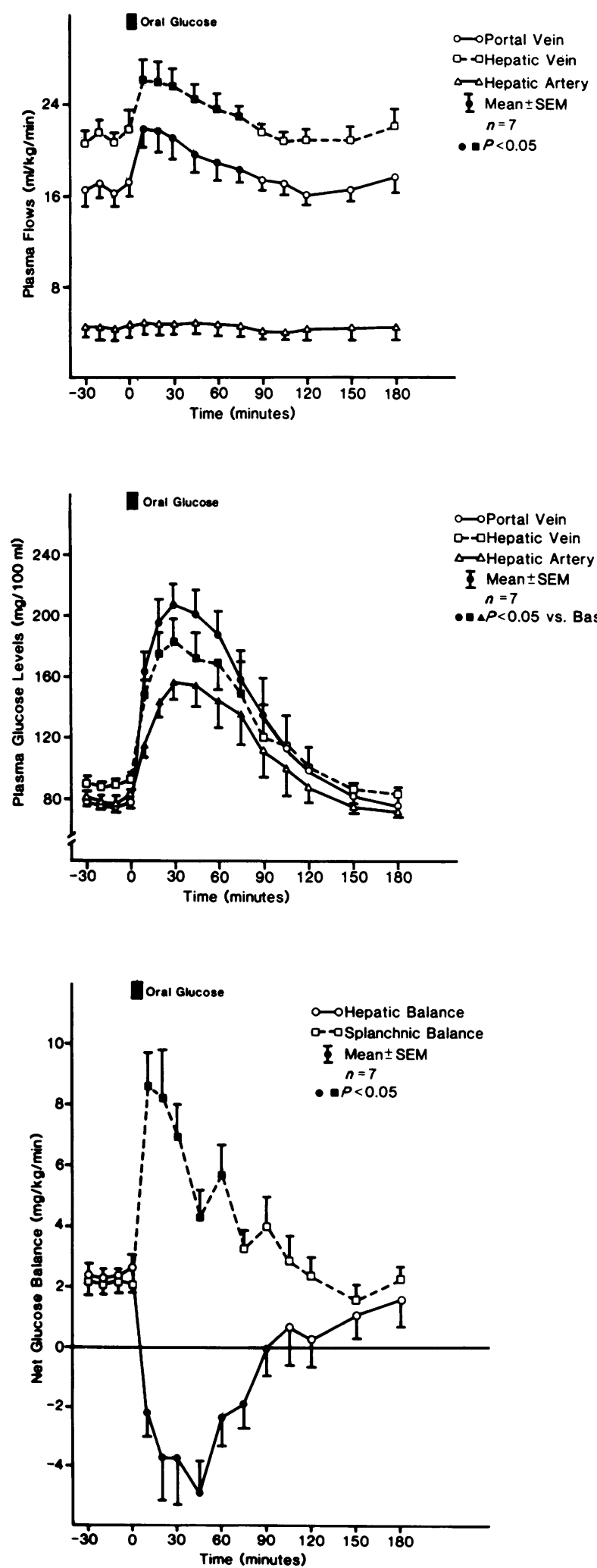

Figure 1. Effect of oral glucose with (right panels) and without (left panels) atropine infusion on portal vein, hepatic vein, and hepatic artery plasma flow (upper panels), plasma glucose concentrations in the
Blood flows in the portal vein and hepatic artery were measured continually (3). They were corrected to plasma flow based on hematocrits obtained every $30 \mathrm{~min}$, since glucose and insulin were measured in plasma. Saline was infused into the cephalic vein to compensate for blood loss.
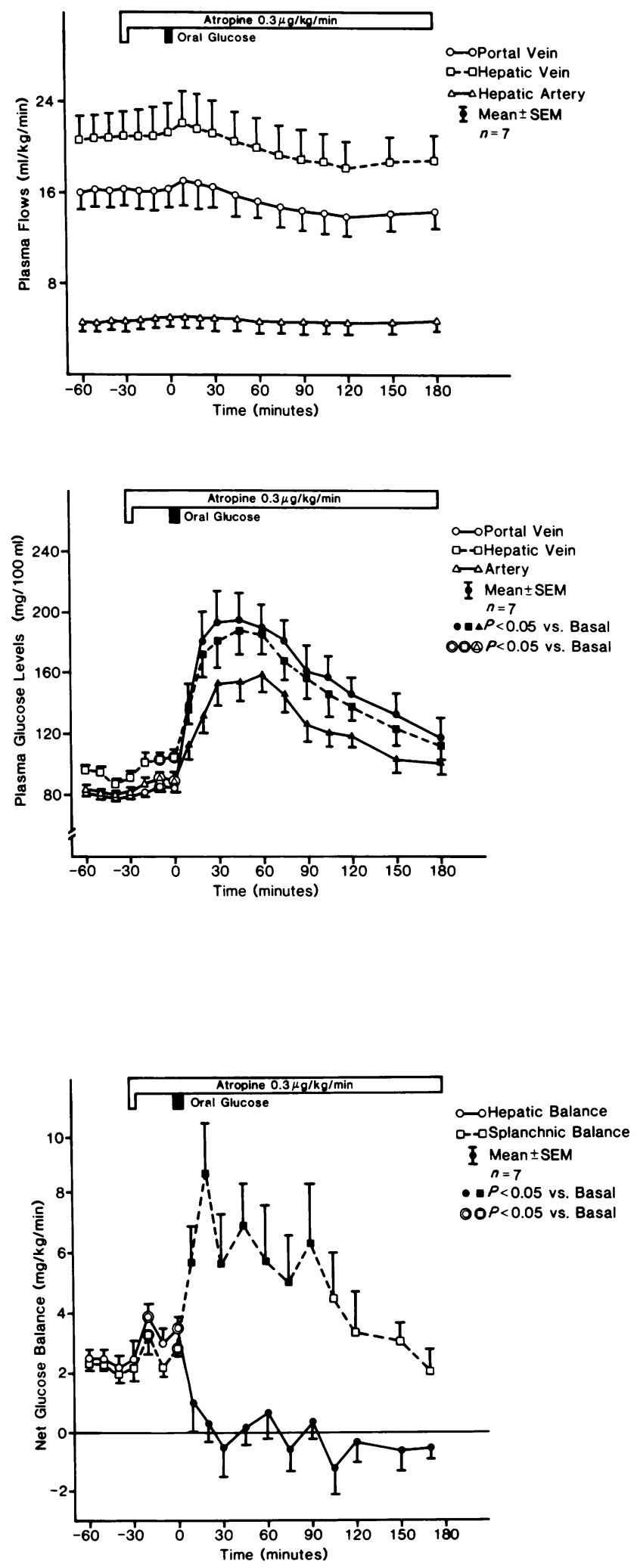

portal vein, hepatic vein, and carotid artery (middle panels), and net hepatic and splanchnic glucose balance (lower panels) 


\section{Experimental procedures}

Oral glucose administration. After a 30-min control period, seven dogs consumed glucose $(1.1 \pm 0.1 \mathrm{~g} / \mathrm{kg}$ per body weight) as a $10 \%$ glucose solution in 2 min. Blood samples were obtained at $-30,-20,-10,0,10$, $20,30,45,60,75,90,105,120,150$, and $180 \mathrm{~min}$. In another seven dogs after a 30-min control period ( $-60--30 \mathrm{~min})$, atropine was infused into the jugular vein from -30 to $180 \mathrm{~min}$ at a rate of $0.38 \mathrm{mg} / \mathrm{h}$ after a bolus injection $(100 \mu \mathrm{g})$ at $-30 \mathrm{~min}$. Glucose $(1.9 \pm 0.1 \mathrm{~g} / \mathrm{kg}$ body weight $)$ was given as a $10 \%$ solution orally at zero time and consumed in $2 \mathrm{~min}$. Since atropine delayed and reduced glucose absorption, the glucose load was increased in order to match the portal vein glucose and insulin con-
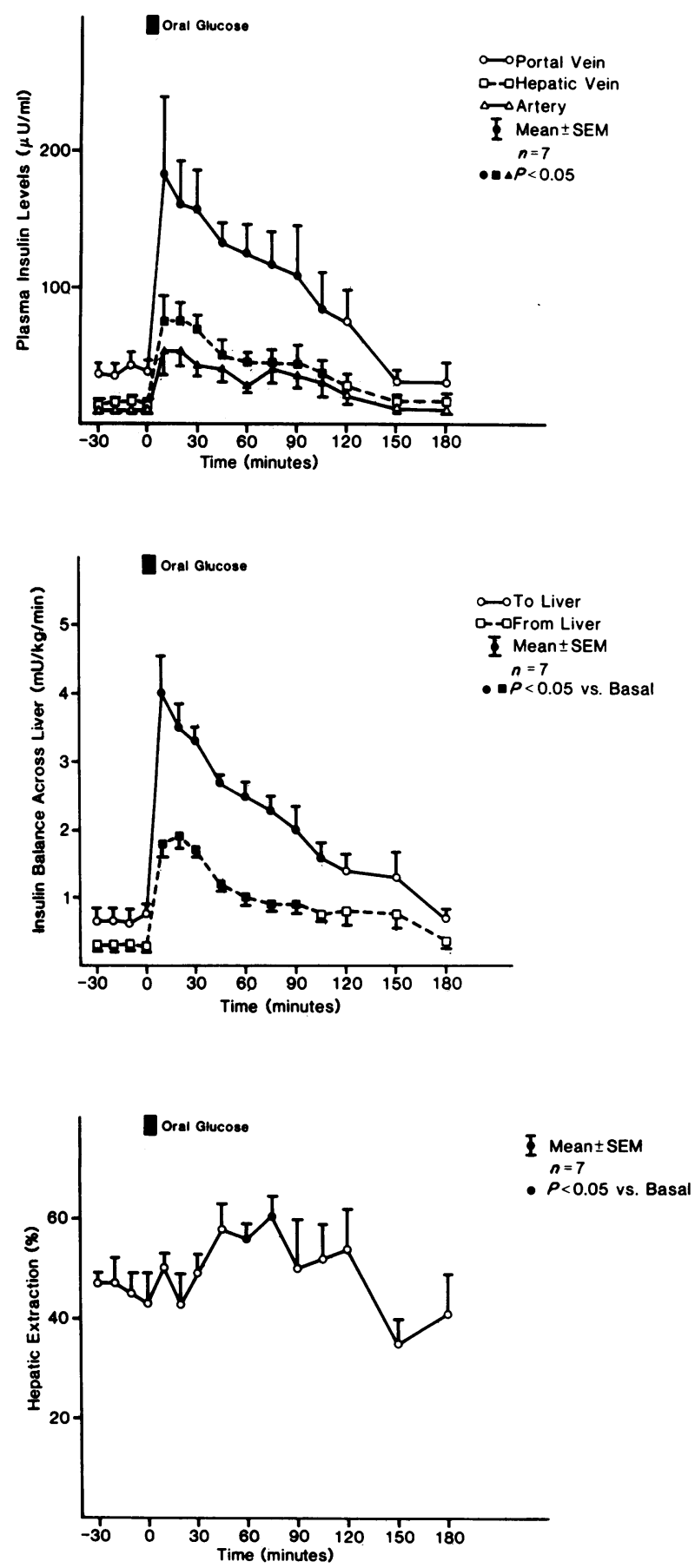

Figure 2. Effect of oral glucose with (right panels) and without (left panels) atropine infusion on plasma insulin concentrations in the portal vein, hepatic vein, and carotid artery (upper panels), insulin bal- centrations that were achieved after oral glucose without atropine. Blood samples were obtained every $10 \mathrm{~min}$ from -60 to $0 \mathrm{~min}$, and then at the same times as in the experiments with oral glucose without atropine.

Peripheral intravenous glucose administration with GIP. After a 30min control period (-30-0 min), GIP obtained from the National Institute of Arthritis, Diabetes, Digestive and Kidney Diseases was infused into the superior mesenteric vein in eight dogs from 0 to $60 \mathrm{~min}$ at a rate of $20 \mathrm{ng} / \mathrm{kg}$ per min. Glucose (5\% in water) was also infused into the jugular vein at the following rates: $0.8 \mathrm{mg} / \mathrm{kg}$ per min from 0 to 10 min, $1.3 \mathrm{mg} / \mathrm{kg}$ per min from 10 to $60 \mathrm{~min}, 0.8 \mathrm{mg} / \mathrm{kg}$ per min from 60 to $75 \mathrm{~min}, 0.4 \mathrm{mg} / \mathrm{kg}$ per $\mathrm{min}$ from 75 to $90 \mathrm{~min}$, and $0.3 \mathrm{mg} / \mathrm{kg}$ per
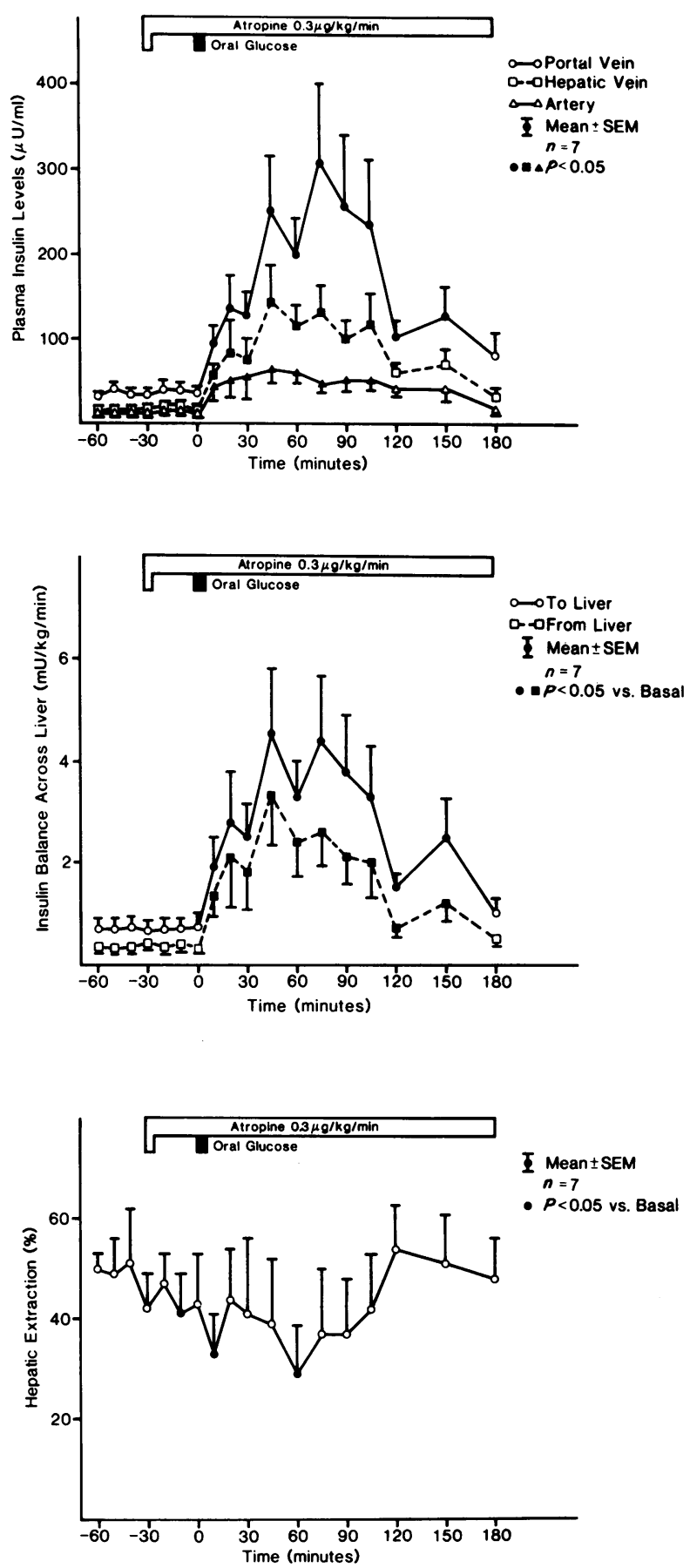

ance across the liver (middle panels), and fractional hepatic extraction of insulin (lower panels). 
min from 90 to $120 \mathrm{~min}$. This rate was the same as that infused intraportally to match the portal vein glucose concentrations after oral glucose as previously reported (3). The control experiments for the dogs infused with GIP and peripheral glucose have been published (3), but are included for easier comparison. Blood samples were obtained at the same times as after oral glucose. Insulin (3), glucagon (3), and GIP (6) were assayed by previously reported methods.

The flux of glucose and hormone in each vessel was obtained by multiplying the plasma concentration by the plasma flow in that vessel. Hepatic vein plasma flow was the sum of the portal vein and hepatic artery plasma flows. The amount of glucose and insulin presented to the liver was the sum of the contribution from the portal vein and hepatic artery (concentration times flow). The amount leaving the liver was theproduct of hepatic vein plasma flow and hepatic vein concentration. The fractional hepatic extraction of insulin was calculated as follows: (insulin presented to the liver - insulin leaving the liver)/(insulin presented to the liver) $\times 100 \%$. The net hepatic balance of glucose was obtained by subtracting the amount of glucose presented to the liver from the amount leaving that organ. Positive values indicate net hepatic glucose output, while negative numbers represent net hepatic glucose uptake. In some cases, these values have been integrated over time as the area under the curve. The net absorption of glucose was calculated by multiplying the portal vein-arterial glucose concentration by the portal vein plasma flow.
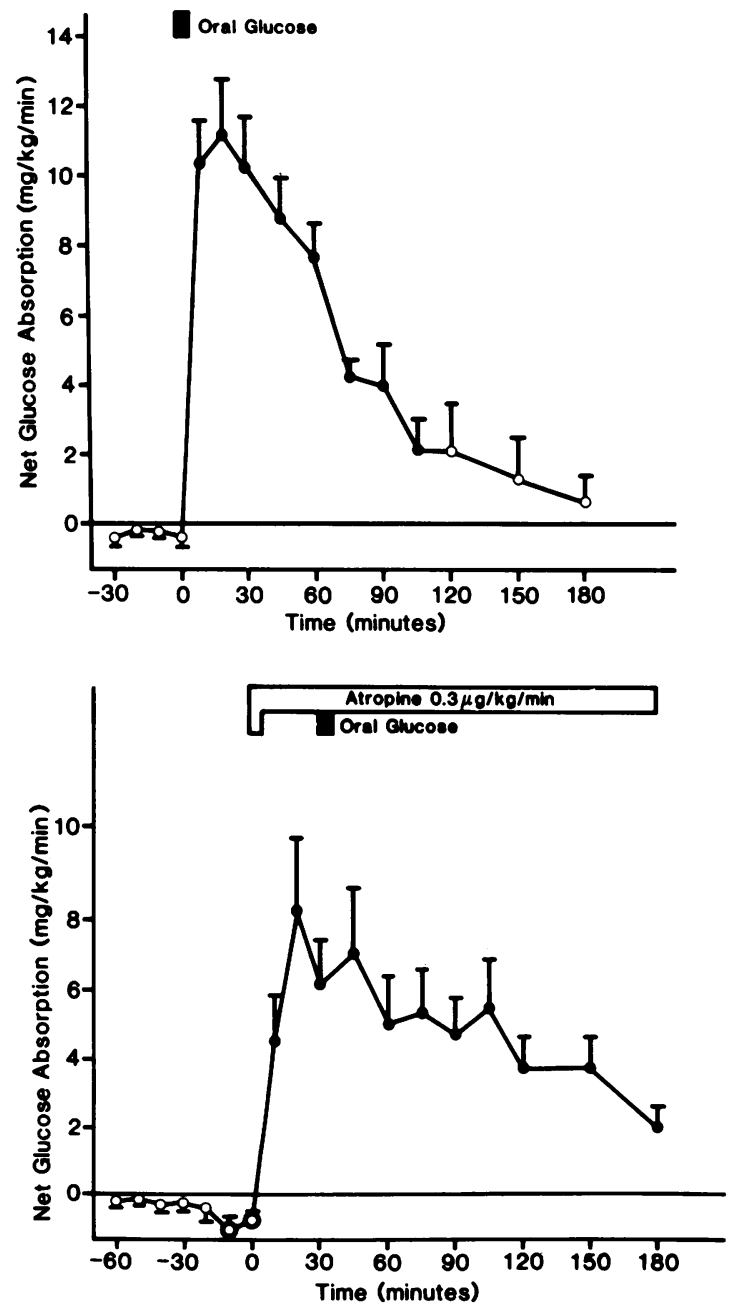

Figure 3. Effect of oral glucose with (bottom) and without (top) atropine infusion on net glucose absorption. The method of calculation of net glucose absorption is given in the Methods section. (Top) $\bullet$, Mean \pm SEM, $n=7 ; \bullet, P<0.05$ vs. basal. (Bottom) $\odot, P<0.05$ vs. basal. Other symbols same as top.
The data are presented as means \pm SEM. The basal value was the mean \pm SEM of the four values obtained from -30 to $0 \mathrm{~min}$. Paired $t$ test was employed for statistical analyses of the change from the basal value within a group. Differences in mean values between groups were detected by unpaired $t$ test. $P$ values $<0.05$ were considered to be significant.

\section{Results}

Atropine infusion before the administration of oral glucose had no effect on basal portal vein plasma flow (Fig. 1, upper right panel). However, it significantly increased net hepatic glucose production after 10 and 30 min of infusion, which resulted in a significant increase in plasma glucose concentration in all three vessels (Fig. 1, middle right panel). There was no significant change in the basal portal vein insulin concentration (Fig. 2, upper panels) or fractional hepatic extraction of insulin (Fig. 2, lower panels). However, infusion of atropine inhibited the significant rise in portal vein plasma flow, which was observed after oral glucose (Fig. 1, upper panels). The increase and peak glucose concentration in the portal vein was similar in both groups, but was slightly, but not statistically delayed and more prolonged in the dogs infused with atropine (Fig. 1, middle panels). The amount of glucose absorbed into the portal system was similar
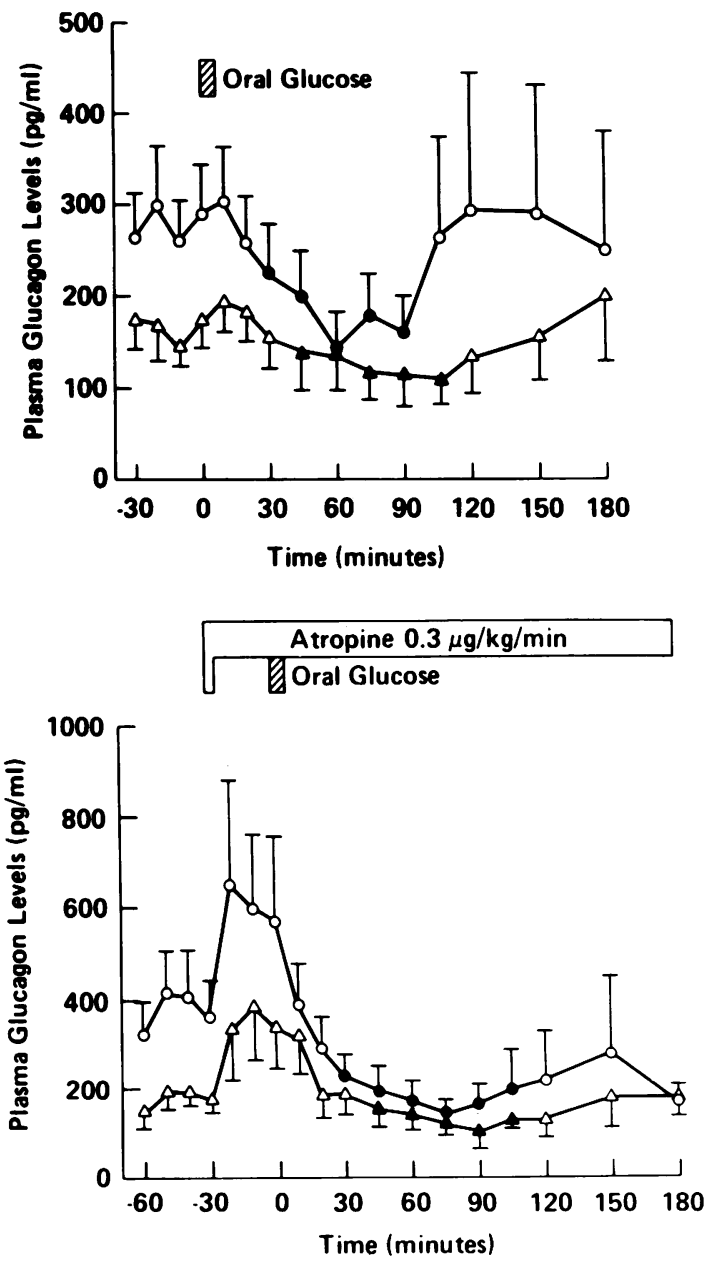

Figure 4. Effect of oral glucose with (bottom) and without (top) atropine infusion on portal vein and arterial glucagon concentrations. $O$, Portal vein; $\Delta$, artery; $\perp$, mean \pm SEM, $n=6$ (top), $n=7$ (bottom); $\bullet$, $\triangle, P<0.05$ vs. basal. 
in both groups of dogs $(20.2 \pm 1.6 \mathrm{~g}$ in control and $21.7 \pm 4.1 \mathrm{~g}$ in atropine dogs) (Fig. 3). This was achieved by administering a larger amount of glucose to the dogs receiving the atropine infusion. Thus, while $80 \pm 4 \%$ of the $1.1 \pm 0.1 \mathrm{~g} / \mathrm{kg}$ glucose given to the control dogs was accounted for in the portal system, only $44 \pm 7 \%$ of the $1.9 \pm 0.1 \mathrm{~g} / \mathrm{kg}$ glucose administered to the atropine dogs was found in the portal system over the 3 -h period. The net hepatic glucose uptake was significantly less at 10,20 , and
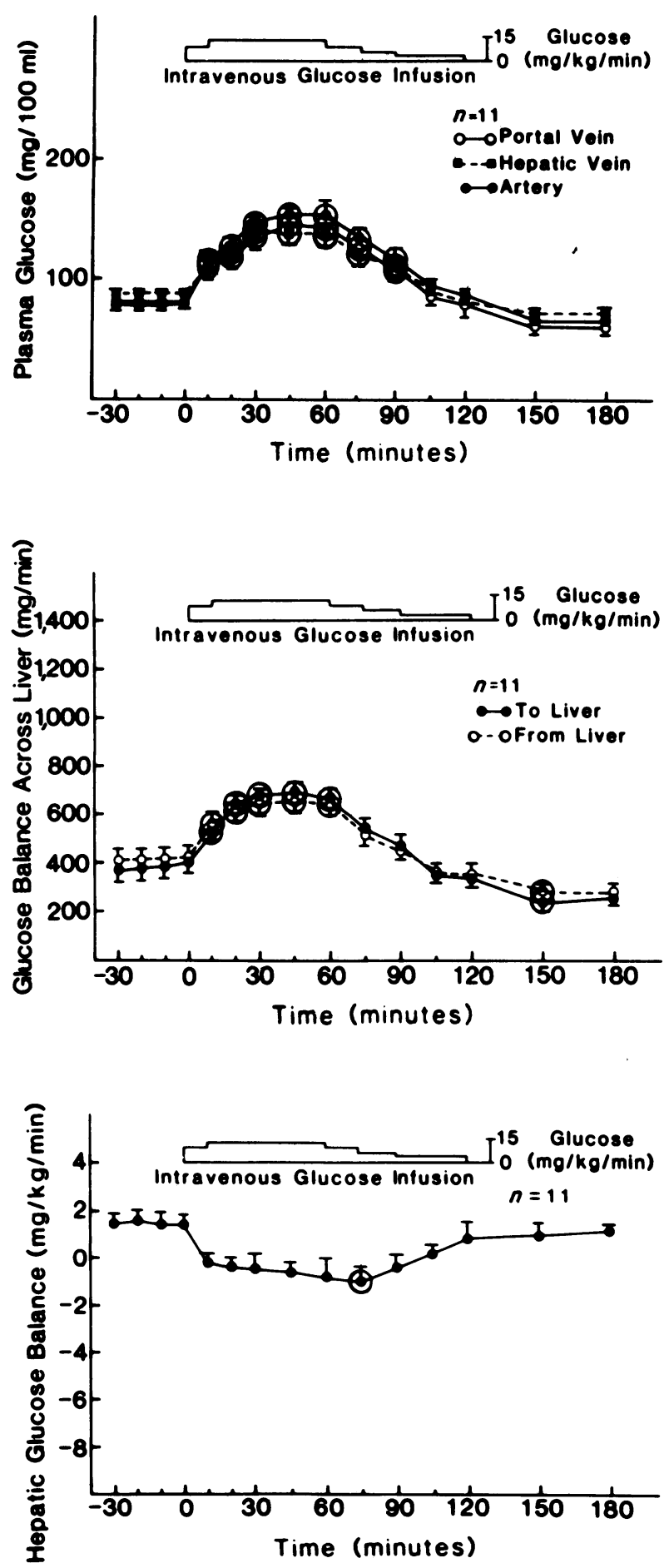

Figure 5. Effect of intraportal GIP and peripheral intravenous glucose infusion on plasma glucose (upper panels), glucose balance across the liver (middle panels), and net hepatic glucose balance (lower panels).
$45 \mathrm{~min}$ in the dogs infused with atropine compared with the control dogs (Fig. 1, lower panels), while the splanchnic glucose output was similar in both groups. Although the shape of the curve was different, net glucose uptake as assessed by the area under the curve over 180 min was not statistically different in the two groups $(5.6 \pm 1$ vs. $3.2 \pm 0.9 \mathrm{~g}$ ). After oral glucose, glucose uptake was greater and was no longer present after $90 \mathrm{~min}$, while after atropine it persisted for the remainder of the experiment.
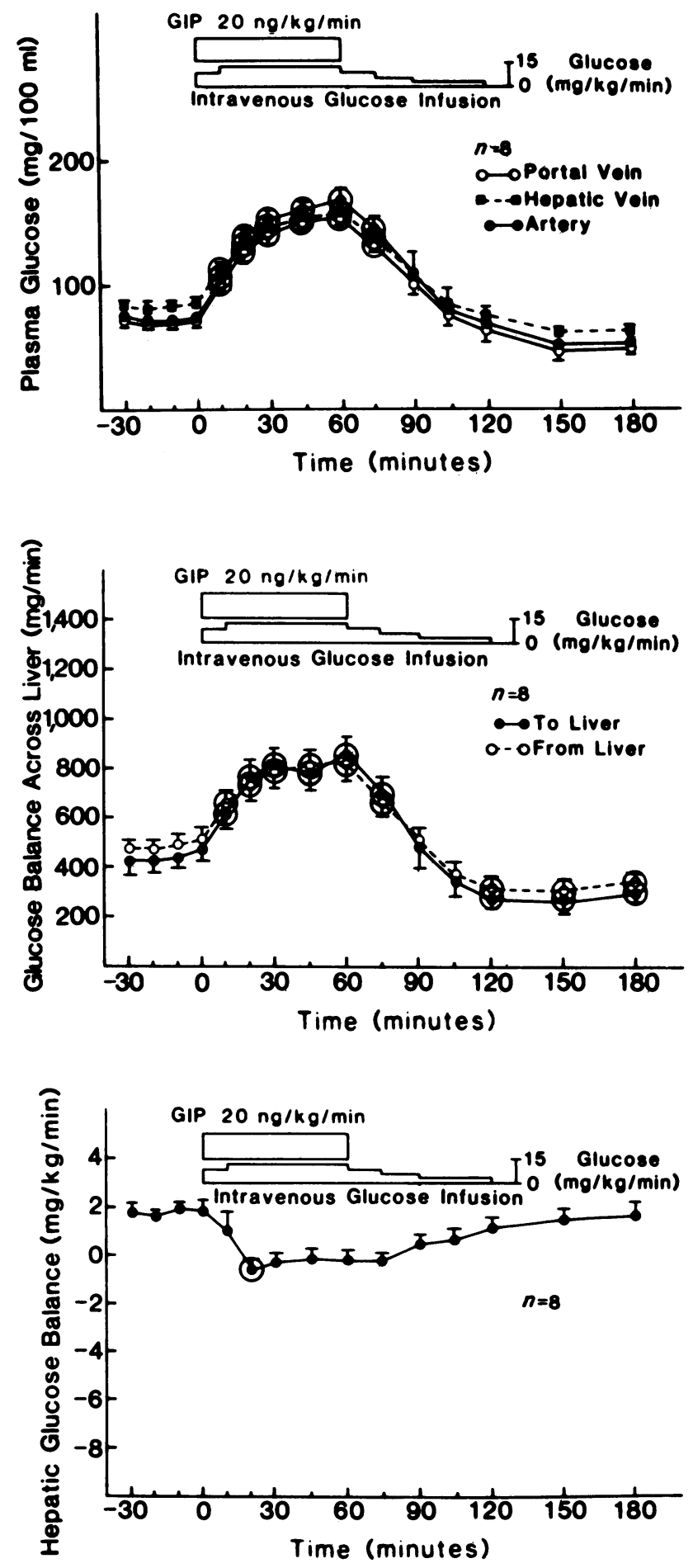

The data from the animals infused with glucose without GIP (left panels) have been published previously (3). 
Infusion of atropine was associated with a delay in the increase in portal vein insulin concentrations compared with oral glucose alone (Fig. 2, upper panels), but the values eventually achieved were greater than after glucose alone. The delay in the rise in portal vein insulin concentrations reflected the slower increase in arterial glucose values after atropine and oral glucose. The insulin concentrations in the hepatic vein at 60 and $75 \mathrm{~min}$ and artery at $60 \mathrm{~min}$ were significantly greater in the atropine dogs compared with the controls (Fig. 2, upper panels). Basal fractional hepatic extraction of insulin was similar in both groups of dogs and was not influenced by atropine infusion (Fig. 2, lower panels). Oral administration of glucose was associated with a significant increased fractional hepatic extraction of insulin, in confirmation of previous results (1-3). In contrast, despite similar amounts of insulin and glucose presented to the liver after oral glucose and infusion of atropine, no significant increase in the fractional hepatic extraction of insulin was observed, and, in fact, the values at 10 and 60 min were significantly less than the control. During the infusion of atropine alone, there was a significant $(P<0.05)$ increase in the plasma glucagon concentrations when the average value during atropine was compared with that before its infusion (Fig. 4). Atropine did not modify the decline of plasma glucagon levels induced by oral glucose (Fig. 4).

Peripheral infusion of glucose produced similar arterial and hepatic vein glucose concentrations to those achieved after oral glucose, but the portal vein glucose concentrations were significantly less in the former situation (3) (Fig. 1, middle left panel, and Fig. 5, upper left panel). However, infusion of GIP into the portal system to mimic the increase observed after oral glucose (Fig. 6) had no apparent effect on the glucose concentration in the three vessels during peripheral intravenous infusion of glucose (Fig. 5, upper right panel). As reported previously (3), the net hepatic glucose uptake was significantly less after peripheral intravenous glucose administration compared with oral glucose (Fig. 1, lower left panel, and Fig. 5, lower left panel), and was not increased by the concomitant infusion of GIP into the portal circulation. Although GIP did not modify net hepatic uptake of glucose after its peripheral intravenous administration, it did have a biologic effect, in that it augmented pancreatic insulin

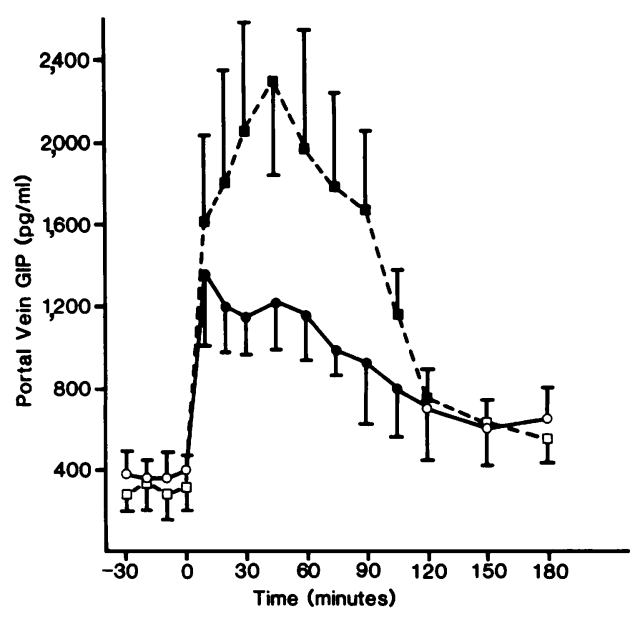

Figure 6. Portal vein GIP concentrations after oral glucose compared with those achieved with intraportal GIP and peripheral vein glucose infusion. ०, Oral glucose; $\square$, intravenous glucose plus GIP; $\bullet$, mean \pm SEM, $n=6 ; \bullet, \bullet, P<0.05$ compared with basal. secretion in response to the hyperglycemia (Fig. 7, upper panels). Thus, after GIP, the amount of insulin delivered to the liver increased to $95 \pm 23 \mathrm{mU} / \mathrm{min}$ at $60 \mathrm{~min}$, compared to $60 \pm 4 \mathrm{mU} /$ min with peripheral intravenous infusion of glucose without GIP. GIP did not modify the suppression of glucagon after the intravenous infusion of glucose (Fig. 7, middle panels), and had no effect on the fractional hepatic extraction of insulin (Fig. 7, lower panels).

\section{Discussion}

The mechanism for the augmented fractional hepatic extraction of insulin after oral glucose (1-3) compared with peripheral intravenous glucose infusion (3) is not known. We have previously discussed the relationship between the amount of glucose presented to the liver and fractional hepatic extraction of insulin and the evidence that the former does not determine the latter (3). The present results with atropine provide additional support for this conclusion. Thus, despite equivalent amounts of glucose reaching the liver after oral glucose in the two experiments, atropine inhibited the expected increased fractional hepatic extraction of insulin (Fig. 2, lower panels). Since atropine delayed and decreased the absorption of glucose, it was necessary to administer a significantly larger amount of glucose to these dogs to achieve comparable portal vein concentrations of glucose in the two groups (Fig. 1, middle panels). This was achieved as indicated both by the actual glucose concentrations in the portal vein as well as by the amount of glucose absorbed over the 3-h experimental period (Fig. 3). Since the rate at which the portal vein glucose concentration increased was more rapid without atropine, it is possible that this could be an important factor in the augmented fractional hepatic extraction of insulin. However, our previous experiments in which oral and intraportal glucose were administered make this less likely (3). Thus, despite the same amounts and rates of glucose presented to the liver, increased fractional hepatic extraction of insulin was observed only after oral glucose. Since atropine infusion did not decrease, and may have actually increased the insulin response to oral glucose (Fig. 2, middle panels), it is unlikely that changes in the amount of insulin presented to the liver are important in the augmented fractional hepatic extraction of insulin after oral glucose. Experiments in which different amounts of insulin were infused into the portal circulation also indicated that insulin was not an important determinant of its fractional hepatic extraction (4).

Several other possibilities must be considered to explain the inhibition by atropine of the increased fractional hepatic extraction of insulin. These include the role of increased portal vein plasma flow and the secretion of gut factors. Since atropine inhibited both the increased portal vein plasma flow (Fig. 1, upper panels) and augmented fractional hepatic extraction of insulin after oral glucose (Fig. 2, lower panels), the former may be an important determinant of the latter. Such a relationship is consistent with our previous observation that ingestion of meat increased both portal vein plasma flow and fractional hepatic extraction of insulin (7). However, infusion of arginine and cholecystokinin also significantly increased portal vein plasma flow, but was associated with a significant decrease in fractional hepatic extraction of insulin (8). Conflicting results have been obtained in the isolated perfused liver regarding the effect of flow on hepatic extraction of insulin $(9,10)$. Although the present experiments do not permit a conclusion concerning the role of portal vein plasma flow in fractional hepatic extraction of insulin, they 

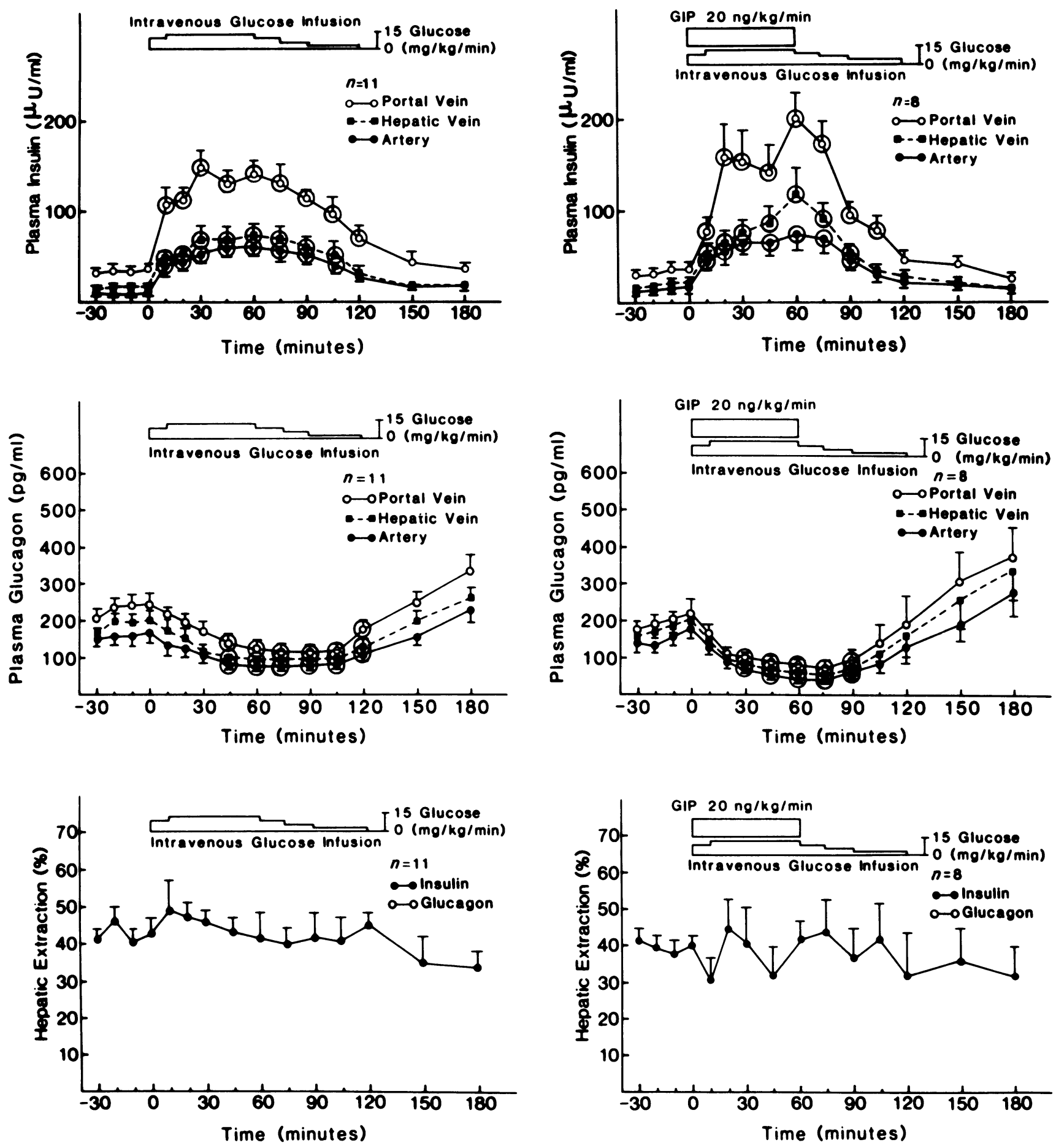

Figure 7. Effect of intraportal GIP and peripheral intravenous glucose infusion on plasma insulin (upper panels), plasma glucagon (middle panels), and fractional hepatic extraction of insulin (lower panels). The

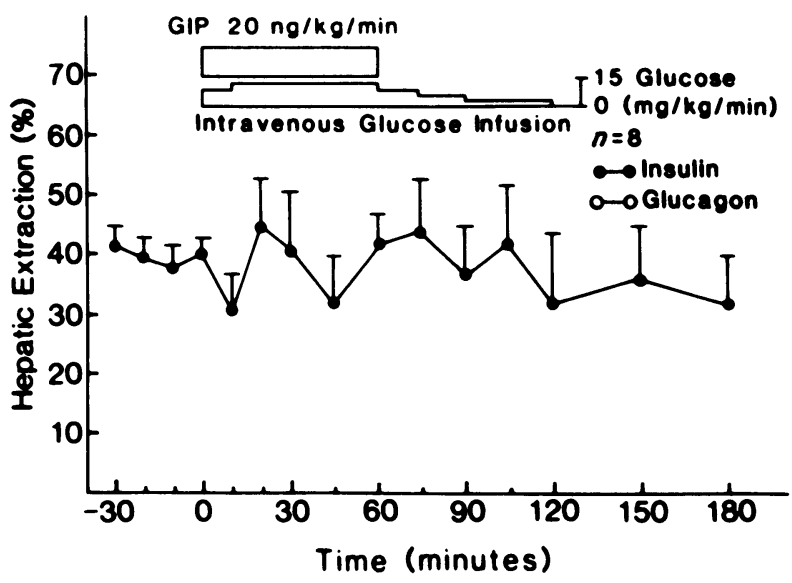

data from the animals infused with glucose without GIP (left panels) have been published previously (3).

provide information related to the mechanism of such increased plasma flow after oral glucose. Increased portal vein flow does not directly reflect the portal vein hyperglycemia or the amount of glucose absorbed, since these were similar in both groups of dogs. The present studies, however, cannot exclude the rate of absorption as being important, since this was delayed by atropine (Fig. 3). Inhibition of the increased portal flow by atropine suggests that it is dependent upon an intact muscarinic parasympathetic innervation. It could be a direct parasympathetic effect,

or it could be mediated by a release of gut factors in response to ingestion of nutrients. The failure of portal infusion of GIP with peripheral infusion of glucose to increase fractional hepatic extraction of insulin (Fig. 7, lower right panel, compared with Fig. 2, lower left panel) confirm the previous results of Polonsky et al. (11) and indicate that this gut hormone is not involved in that phenomenon. The portal vein GIP levels obtained during its infusion were actually greater than those achieved after oral glucose (Fig. 6), and the biologic effectiveness of the GIP is in- 
dicated by its potentiation of insulin secretion after peripheral intravenous infusion of glucose (Fig. 7, upper panels). These results do not preclude the involvement of other gut hormones in the increased fractional hepatic extraction of insulin.

The present results provide additional information concerning the greater hepatic glucose uptake after oral and intraportal glucose compared with peripheral intravenous glucose (3). It seems unlikely that the greater uptake of glucose by the liver after oral glucose (Fig. 1, lower panels) is due to the augmented fractional hepatic extraction of insulin (3). Thus, hepatic glucose uptake was similar after both oral and intraportal glucose administration $(3,5)$, but only the former was associated with increased fractional hepatic extraction of insulin. Whether atropine decreased the greater hepatic glucose uptake after oral glucose depends upon how the data is evaluated. It significantly diminished it at 10, 20, and $45 \mathrm{~min}$, while there was no significant difference when it was calculated as the area under the curve. If atropine does not decrease the hepatic uptake of glucose, yet inhibited the fractional hepatic extraction of insulin, it indicates that the latter is not necessary for the greater hepatic uptake after oral glucose. However, if atropine did decrease the hepatic uptake of glucose, it still does not necessarily prove that the two are related. The different shapes of the curves of net hepatic glucose uptake with and without atropine may reflect several factors. First, atropine itself increased hepatic glucose production (Fig. 1, lower panels), and a transient continuation of this effect might affect net hepatic balance. This almost certainly reflects the increase of glucagon in the portal vein during atropine infusion alone (Fig. 4). Secondly, the rate of glucose absorption was slower after atropine. However, these differences may not fully explain the significantly greater net hepatic glucose uptake after oral glucose during the first hour as compared with the uptake with atropine infusion; this is especially true since the peak levels of glucose in the portal vein occurred during this time. Gut (12-15) or neurogenic factors, especially parasympathetic $(16,17)$, may be important in the control of hepatic glucose uptake. Thus, the inhibition by atropine of the augmented net hepatic glucose uptake during the first hour after oral glucose could reflect inhibition of neurogenic or gut factors. It is unlikely that GIP is responsible for the augmented hepatic uptake of glucose, since its administration (which resulted in portal vein GIP concentrations greater than after oral glucose [Fig. 6]) with peripherally infused glucose did not increase hepatic uptake of glucose or reproduce the effect obtained with oral or intraportal glucose (3). A role for the parasympathetic nervous system is also suggested by the results of Mondon and Burton (16), who reported that pharmacologic amounts of acetylcholine in the presence of insulin markedly enhanced the uptake of glucose by the isolated perfused rat liver. In addition, electrical stimulation of the parasympathetic nerves isolated from around the common hepatic artery rapidly suppressed hepatic glucose output (17). Our finding that atropine significantly reduced the net hepatic glucose uptake after oral glucose during the first hour suggests the possibility that a muscarinic parasympathetic action is responsible for this change in hepatic glucose metabolism.

\section{Acknowledgments}

The authors are indebted to the staff of the Cardiovascular Sciences Section for their superb help in the execution of these experiments. Dr.
Thomas O'Dorisio and Dr. Samuel Cataland, Department of Medicine, Ohio State University School of Medicine, Columbus, $\mathrm{OH}$, kindly provided the assays of GIP. We would also like to thank Mrs. Shelley Dearing and Ms. Barbara Sims for their outstanding assistance in the preparation of this manuscript.

This work was supported by U. S. Public Health Service grants AM 25253 and AM 27685 from the National Institutes of Health.

\section{References}

1. Kaden, M., P. Harding, and J. B. Field. 1973. Effect of intraduodenal glucose administration on hepatic extraction of insulin in the anesthetized dog. J. Clin. Invest. 52:2016-2028.

2. Jaspan, J., and K. Polonsky. 1982. Glucose ingestion in dogs alters the hepatic extraction of insulin. In vivo evidence for a relationship between biologic action and extraction of insulin. J. Clin. Invest. 69:516525.

3. Ishida, T., Z. Chap, J. Chou, R. Lewis, C. Hartley, M. Entman, and J. B. Field. 1983. Differential effects of oral, peripheral intravenous and intraportal glucose on hepatic glucose uptake and insulin and glucagon extraction in conscious dogs. J. Clin. Invest. 72:590-601.

4. Harding, P., G. Bloom, and J. B. Field. 1975. Effect of infusion of insulin into portal vein on hepatic extraction of insulin in anesthetized dogs. Am. J. Physiol. 228:1580-1588.

5. Bergman, R. N., J. R. Beir, and P. M. Hourigan. 1982. Intraportal glucose infusion matched to oral glucose absorption. Lack of evidence for "gut factor" involvement in hepatic glucose storage. Diabetes. 31: 27-35.

6. Yovos, J. G., T. O'Dorisio, S. Cataland, F. B. Thomas, H. Mehkjian, and L. O. Carey. 1982. Effects of amino acids and gastric inhibitory polypeptide on insulin release in dogs. Am. J. Physiol. 242:E53-E58.

7. Ishida, T., J. Chou, R. M. Lewis, C. J. Hartley, M. Entman, and J. B. Field. 1983. The effect of ingestion of meat on hepatic extraction of insulin and glucagon and hepatic glucose output in conscious dogs. Metab. Clin. Exp. 32:558-567.

8. Ishida, T., R. M. Lewis, C. J. Hartley, M. L. Entman, and J. B. Field. 1983. Comparison of hepatic extraction of insulin and glucagon in conscious and anesthetized dogs. Endocrinology. 112:1098-1109.

9. Honey, R. N., and S. Price. 1979. The determinants of insulin extraction in the isolated perfused liver. Horm. Metab. Res. 11:111-117.

10. Misbin, R. N., T. J. Merimee, and J. M. Lowenstein. 1976. Insulin removal by isolated perfused rat liver. Am. J. Physiol. 230:171-177.

11. Polonsky, K., J. Jaspan, W. Pugh, D. Cohen, M. Schneider, T. Schwartz, A. R. Moosa, H. Tager, and A. R. Rubenstein. 1983. The metabolism of C-peptide in the dog: in vivo demonstration of the absence of hepatic extraction. J. Clin. Invest. 72:1114-1123.

12. DeFronzo, R. A., E. Ferrannini, R. Hendler, J. Wahren, and P. Felig. 1978. Influence of hyperinsulinemia, hyperglycemia and the route of glucose administration on splanchnic glucose exchange. Proc. Natl. Acad. Sci. USA. 75:5173-5177.

13. DeFronzo, R., E. Ferrannini, J. Wahren, and P. Felig. 1978. Lack of gastrointestinal mediator of insulin action in maturity-onset diabetes. Lancet. II:1077-1079.

14. Dupre, J. 1964. An intestinal hormone affecting glucose disposal in man. Lancet. II:672-673.

15. Anderson, D. K., W. S. Putnam, J. B. Hanks, J. E. Wise, H. E. Lebovitz, and R. S. Jones. 1980. Gastric inhibitory polypeptide (GIP) suppression of hepatic glucose production. Regul. Pept. 1(Suppl. 1):S4. (Abstr.)

16. Mondon, C. E., and S. D. Burton. 1971. Factors modifying carbohydrate metabolism and effect of insulin in perfused rat liver. Am. J. Physiol. 220:724-734.

17. Lautt, W. W., and C. Wong. 1978. Hepatic parasympathetic neural effect on glucose balance in the intact liver. Can. J. Physiol. Pharmacol. 56:679-682. 